\title{
ON THE COMPUTATION OF ZEROES OF $J_{n}(z)-i J_{n+1}(z)=0$
}

BY

\author{
D. A. MACDONALD
}

Department of Applied Mathematics and Theoretical Physics, The University, Liverpool

Abstract. The roots of the equation

$$
J_{n}^{2}(z)+J_{n+1}^{2}(z)=0
$$

in which $n$ is a positive integer or zero, are of interest to the specialist in wave reflection from multi-sloped beaches [1]. This note shows how to obtain accurate roots of the equation when $n$ is not large.

1. Introduction. The equation $F_{n}(z)=0$, where

$$
F_{n}(z)=J_{n}^{2}(z)+J_{n+1}^{2}(z)
$$

and $n$ is a positive integer or zero, has no real or purely imaginary roots. Since $F_{n}(-z)=$ $F_{n}(z)$ and $\overline{F_{n}(z)}=F_{n}(\bar{z})$, whenever $z_{k}$ is a root so too are $-z_{k}$ and $\pm \bar{z}_{k}$. Hence, when calculating the roots it is sufficient to confine attention to any one quadrant of the $z$ plane. The problem can be simplified by observing that

$$
F_{n}(z)=\left(J_{n}(z)+i J_{n+1}(z)\right)\left(J_{n}(z)-i J_{n+1}(z)\right) .
$$

Hence the roots of (1) can be found by solving either one of

$$
\begin{aligned}
& J_{n}(z)+i J_{n+1}(z)=0, \\
& J_{n}(z)-i J_{n+1}(z)=0 .
\end{aligned}
$$

Since the roots of $J_{n}(z)=0$ are all real ([2], §15.25) no root can satisfy both Eqs. (2) and (3). Indeed it has been shown, [1], that the roots of (2) are confined to the upper half of the $z$ plane whereas those of (3) all lie in the lower half plane.

2. Computation of roots. Three methods will be used to evaluate approximations to the roots of

$$
J_{n}(z)-i J_{n+1}(z)=0
$$

Received October 6, 1994.

1991 Mathematics Subject Classification. Primary 33C10, 41A60. 
Method A is analytic and leads to asymptotic formulae for the roots. Methods B and C are numerical and rely on Newton-Raphson iteration: B is strictly valid when $|z| \gg 1$ whereas, due to rounding error difficulties (see $\S 2.3$ ), $\mathrm{C}$ is valid for $|z|$ not too large.

2.1. Method A: The asymptotic formulae. As in [3], we choose to work with

$$
I_{n}(\zeta)+I_{n+1}(\zeta)=0
$$

where $z=i \zeta$, rather than (3). Since the roots of (3) lie in the lower half plane, $-\pi<$ $\arg z<0$. Hence $-\frac{3 \pi}{2}<\arg \zeta<-\frac{\pi}{2}$ and $([2], \S 7.23)$

$$
I_{n}(\zeta) \sim \frac{e^{\zeta}}{\sqrt{2 \pi \zeta}}\left[1+\sum_{m=1}^{\infty}(-1)^{m} \frac{(n, m)}{(2 \zeta)^{m}}\right]-i(-1)^{n} \frac{e^{-\zeta}}{\sqrt{2 \pi \zeta}}\left[1+\sum_{m=1}^{\infty} \frac{(n, m)}{(2 \zeta)^{m}}\right],
$$

where, for $m=1,2, \ldots$,

$$
(n, m)=\frac{\left(4 n^{2}-1^{2}\right)\left(4 n^{2}-3^{2}\right) \cdots\left(4 n^{2}-[2 m-1]^{2}\right)}{2^{2 m} m !} .
$$

When (5), with each series truncated at its fifth term, is substituted in (4), the logarithm of the resulting expression gives

$$
\begin{aligned}
2 \zeta=-\ln (4 \zeta)+ & \ln (2 n+1)+\left(2 p+n+\frac{3}{2}\right) \pi i-\frac{1}{4 \zeta}\left(1-4 n-4 n^{2}\right) \\
& +\frac{1}{16 \zeta^{2}}\left(5-4 n-4 n^{2}\right)-\frac{1}{192 \zeta^{3}}\left(16 n^{4}+32 n^{3}-104 n^{2}-120 n+73\right) \\
& +\frac{1}{512 \zeta^{4}}\left(48 n^{4}+96 n^{3}-536 n^{2}-584 n+419\right)+O\left(1 /|\zeta|^{5}\right),
\end{aligned}
$$

where $p$ is an integer.

The leading approximation for $\zeta$. For $|\zeta| \gg 1$ and $n$ not large, a first approximation to $\zeta$ may be obtained by solving

$$
2 \zeta=-\ln (4 \zeta)+\ln (2 n+1)+\left(2 p+n+\frac{3}{2}\right) \pi i-\frac{1}{4 \zeta}\left(1-4 n-4 n^{2}\right)+O\left(1 /|\zeta|^{2}\right) .
$$

Write $\zeta=r e^{i \theta}$, where, for the third quadrant roots of (4), $-\pi<\theta<-\frac{\pi}{2}$. Then, from (7),

$$
\cos \theta=-\frac{1}{2 r} \ln \left(\frac{4 r}{2 n+1}\right)+O\left(\frac{1}{r^{2}}\right) .
$$

Hence, for $r \gg 1, \theta$ is close to $-\frac{\pi}{2}$. So, when $r \gg 1$, we write $\theta=-\frac{\pi}{2}+\phi$, where $0<-\phi \ll 1$.

Again, from (7)

$$
\begin{aligned}
& 2 r \sin \phi=-\ln \left(\frac{4 r}{2 n+1}\right)-\frac{\sin \phi}{4 r}\left(1-4 n-4 n^{2}\right)+O\left(\frac{1}{r^{2}}\right) \\
& 2 r \cos \phi=-2 \pi\left(p+1+\frac{n}{2}\right)+\phi+\frac{\cos \phi}{4 r}\left(1-4 n-4 n^{2}\right)+O\left(\frac{1}{r^{2}}\right) .
\end{aligned}
$$

We note that (see (8))

$$
|\phi|=O\left(\frac{\alpha}{r}\right), \quad \alpha=\frac{1}{2} \ln \left(\frac{4 r}{2 n+1}\right) .
$$


Further, from (9),

$$
\begin{array}{r}
2 r=\left[-2 \pi\left(p+1+\frac{n}{2}\right)+\phi+\frac{\left(1-4 n-4 n^{2}\right)}{4 r}\left(1-\frac{\phi^{2}}{2}+\cdots\right)\right]\left(1+\frac{\phi^{2}}{2}+\cdots\right) \\
+O\left(\frac{1}{r^{2}}\right) .
\end{array}
$$

Since $|\phi|=O(\alpha / r)$ and $r=O(-[p+1+n / 2] \pi)$,

$$
2 r=-2 \pi\left(p+1+\frac{n}{2}\right)\left[1+\frac{\phi^{2}}{2}\right]+\phi+\frac{1-4 n-4 n^{2}}{4 r}+O\left(\frac{1}{r^{2}}\right) \text {. }
$$

From (8),

$$
\sin \phi=-\frac{\alpha}{r}\left[1-\frac{1-4 n-4 n^{2}}{8 r^{2}}\right]+O\left(\frac{1}{r^{3}}\right) .
$$

Hence, when $n=0$ or $n=O(1)$,

$$
\phi=-\frac{\alpha}{r}+O\left(\frac{\alpha}{r}\right)^{3}
$$

From Eqs. (10) and (11) it follows that

$$
\begin{aligned}
2 r & =-2 \pi\left(p+1+\frac{n}{2}\right)\left(1+\frac{\alpha^{2}}{2 r^{2}}\right)-\frac{\alpha}{r}+\frac{\left(1-4 n-4 n^{2}\right)}{4 r}+O\left(\frac{1}{r^{2}}\right), \\
\theta & =-\frac{\pi}{2}-\frac{\alpha}{r}+O\left(\frac{\alpha}{r}\right)^{3} .
\end{aligned}
$$

In Eqs. (12) and (13) $p$ must be a negative integer, say $p=-k-1, k>n / 2$, and $\alpha$ must be positive. Hence the leading term, $r_{a}$ say, in the asymptotic approximation to $r$ satisfies $r_{a}=(k-n / 2) \pi>(2 n+1) / 4$. When $n=0$ we need $k>1 /(4 \pi)$, and the root of smallest modulus corresponds to $k=1$. This has been confirmed numerically, [4]. By use of Schläfli's expansion for the product of two Bessel functions, [2], §5.41, Eq. (1) can be expressed as

$$
\frac{(z / 2)^{2 n}}{(n !)^{2}}\left[1+(2 n+1)(n !)^{2} \sum_{m=1}^{\infty} \frac{(-1)^{m}(z / 2)^{2 m}(2 m+2 n) !}{[(n+m) !]^{2}(2 n+m+1) ! m !}\right]=0 .
$$

Clearly, for $n>0, z=0$ is a $2 n$-fold zero of $F_{n}(z)=0$. The remaining roots satisfy alternating infinite series which are such that the absolute values of the coefficients of $z^{2 m}, m=1,2, \ldots$, decrease with increase of $n .^{1}$ This suggests that, for $n=0,1,2, \ldots$, the root of smallest (nonzero) modulus of $F_{n}(z)=0$ will increase with increase of $n$. This conjecture leads to the conclusion that the root of smallest (nonzero) modulus of $F_{n}(z)=0$ corresponds to $p=-n-2$ in (12).

\footnotetext{
${ }^{1}$ Denote $(2 n+1) !(n !)^{2} \times m$ th term of the series by $(-1)^{m}(z / 2)^{2 m} T(n, m)$. Then $\frac{T(n, m)}{T(n+1, m)}=\frac{8 n^{4}+(16 m+32) n^{3}+\left(10 m^{2}+46[m+1]\right) n^{2}+\left(2 m^{3}+17 m^{2}+41 m+28\right) n+m^{3}+6 m^{2}+11 m+6}{8 n^{4}+(8 m+32) n^{3}+(28 m+46) n^{2}+(32 m+28) n+12 m+6}$.
} 
Iterative solution of Eqs. (12) and (13). The leading approximation to Eqs. (12) and (13) gives purely imaginary roots, with

$$
r_{a}=(j+n / 2) \pi
$$

being the modulus of the $j$ th root. The second approximation is, for this exercise, as far as we need to go. It gives the approximation

$$
\begin{aligned}
& r=r_{a}+\frac{\alpha_{a}^{2}-\alpha_{a}-\left(4 n^{2}+4 n-1\right) / 4}{2 r_{a}}+O\left(\frac{1}{r_{a}^{2}}\right), \\
& \theta=-\frac{\pi}{2}-\frac{\alpha_{a}}{r_{a}}+O\left(\frac{\alpha_{a}}{r_{a}}\right)^{3},
\end{aligned}
$$

where

$$
\alpha_{a}=\frac{1}{2} \ln \left(\frac{4 r_{a}}{2 n+1}\right) .
$$

The solution correct to $O\left(\alpha_{a}\left\{\alpha_{a} / r_{a}\right\}^{3}\right)$. This is obtained from (6) by evaluating the third iterate, $r_{c}$, correct to order $1 /\left(r^{4}\right)$ and by employing results like

$$
\begin{aligned}
& \alpha_{b}=\alpha_{a}+\frac{1}{16 r_{a}^{2}}\left[1-4 n-4 n^{2}+4 \alpha_{a}^{2}-4 \alpha_{a}\right]+O\left(\frac{\alpha_{a}}{r_{a}}\right)^{4}, \\
& \frac{1}{r_{b}}=\frac{1}{r_{a}}\left[1-\frac{1}{8 r_{a}^{2}}\left(1-4 n-4 n^{2}+4 \alpha_{a}^{2}-4 \alpha_{a}\right)+O\left(\frac{\alpha_{a}}{r_{a}}\right)^{4}\right] .
\end{aligned}
$$

We obtain, for the $j$ th root of (4),

$$
\begin{aligned}
r= & r_{a}+\frac{\alpha_{a}^{2}-\alpha_{a}-\left(4 n^{2}+4 n-1\right) / 4}{2 r_{a}} \\
& -\frac{1}{384 r_{a}^{3}}\left[f(n)+\alpha_{a} g(n)-\alpha_{a}^{2} h(n)-256 \alpha_{a}^{3}+48 \alpha_{a}^{4}\right]+O\left(\frac{1}{r_{a}^{4}}\right), \\
\theta= & -\frac{\pi}{2}-\frac{\alpha_{a}}{r_{a}} \\
& +\frac{1}{96 r_{a}^{3}}\left[3\left(12 n^{2}+12 n-7\right)-48 \alpha_{a}\left(2 n^{2}+2 n-1\right)-72 \alpha_{a}^{2}+32 \alpha_{a}^{3}\right]+O\left(\frac{\alpha_{a}}{r_{a}}\right)^{5}
\end{aligned}
$$

where

$$
\begin{aligned}
f(n) & =121-240 n-128 n^{2}+224 n^{3}+112 n^{4}, \\
g(n) & =528 n(n+1)-324, \\
h(n) & =480 n(n+1)-360, \\
r_{a} & =(j+n / 2) \pi .
\end{aligned}
$$

When $n=0$ the $O\left(1 / r_{a}^{3}\right)$ terms independent of $\alpha_{a}$ and linearly dependent on $\alpha_{a}$ in (16) do not agree with those given in Eq. (8) of [3]. This is due to an algebraic error in the latter equation. Table (3) demonstrates the improved accuracy of the corrected result given by (16) and (17). 
TABLE 1. Results for the $s$ th iteration of the $r$ th root, $x+i y$, of $J_{5}-i J_{6}=0$, when $J_{5}$ and $J_{6}$ are represented by their asymptotic expansions correct to $O\left(z^{-13 / 2}\right)$.

\begin{tabular}{|l|l|l|l||c|c|c|c|}
\hline$r$ & $s$ & \multicolumn{2}{|c|}{ Approximate root } & $r$ & $s$ & \multicolumn{3}{|c|}{ Approximate root } \\
\hline 1 & 0 & $9.41 .$. & $-0.663 .$. & 10 & 0 & $38.86995 .$. & $-1.3348 .$. \\
\hline & 1 & $9.33241 .$. & $-0.6953 .$. & & 1 & $38.869857315 .$. & $-1.3354043929 .$. \\
\hline & 2 & $9.3329703 .$. & $-0.6944531 .$. & & 2 & 38.869857307368 & -1.335404390740 \\
\hline & 3 & $9.3329721627 .$. & $-0.6944529932 .$. & & 3 & 38.869857307368 & -1.335404390740 \\
\hline & 4 & 9.332972164857 & -0.694452995816 & & & & \\
\hline & 5 & 9.332972164855 & -0.694452995821 & & & & \\
\hline
\end{tabular}

2.2. Method B: The asymptotic calculation. By use of the asymptotic expansions for $I_{n}(\zeta)$ and $I_{n+1}(\zeta),{ }^{2}(4)$ can be replaced by the $m$ th order asymptotic approximation

$$
e^{\zeta} \sum_{k=0}^{m} a_{n, k} \zeta^{-k}+i e^{-\zeta} \sum_{k=1}^{m} b_{n, k} \zeta^{-k}=0
$$

where the $a_{n, k}$ and the $b_{n, k}$ are constants. For $m=6$ and $n=0,1,2,3,5$, this equation has been solved by Newton-Raphson iteration, with initial approximation given by (16) and (17). In all cases studied, convergence correct to at least 12 places after the decimal point was achieved within five iterations. Table (1) shows the rate of convergence for the first and the tenth roots of $J_{5}(\zeta)-i J_{6}(\zeta)=0$.

2.3. Method $C$ : The ascending series calculation. When each of $I_{n}(\zeta)$ and $I_{n+1}(\zeta)$ is replaced by its ascending series, (4) becomes

$$
\sum_{k=0}^{\infty} \frac{(\zeta / 2)^{n+2 k}}{k !(k+n) !}\left[1+\frac{(\zeta / 2)}{(k+n+1)}\right]=0 .
$$

When (19) is truncated at $k=L$ we obtain the $(n+2 L+1)$ th polynomial equation

$$
\sum_{k=0}^{2 L+1} c_{k}(\zeta / 2)^{n+k}=0
$$

where, for $k=0,1, \ldots, L$

$$
c_{2 k}=\frac{1}{k !(n+k) !}, \quad c_{2 k+1}=\frac{1}{k !(n+1+k) !} .
$$

Now the asymptotic formulae show that for $j \gg 1,\left|\zeta_{j}\right| \sim(j+n / 2) \pi$. Hence, when $j \gg 1$, our polynomial equation has a sum that is zero and certain individual terms that are large in modulus. ${ }^{3}$ Such ingredients are, of course, a standard recipe for rounding error difficulties. This feature of the calculation places an upper bound on the number of roots - $N$, say-of (4) that can be obtained by use of the ascending series approach. Clearly $N=N(D)$, where $D$ denotes the number of digits retained in our Maple (computer algebra) calculation.

For given $D$ and suitably small $|\zeta|$, Eq. (20) can be solved by Newton-Raphson iteration, using as initial approximation the asymptotic formulae of $\S 2.1$, or, for more rapid

\footnotetext{
${ }^{2}$ Or by use of Lommel's polynomials and the asymptotic expansions for $I_{0}(\zeta)$ and $I_{1}(\zeta)$.

${ }^{3}$ When $n=5$ the modulus of the eighteenth root of $I_{5}(\zeta)+I_{6}(\zeta)=0$ is approximately 64 .
} 
convergence, the results of $\S 2.2$. For $n=0,1,2,3,5$, the leading roots of (4) were evaluated, accuracy being checked by repeating the calculations for different values of $L$ and $D$. From (19), a necessary condition for accuracy of the $j$ th root, $\zeta_{j}=r_{j} e^{i \theta_{j}}$, is that

$$
\frac{\left(r_{j} / 2\right)^{2 L+n+1}}{(L+n+1) !(L !)} \ll 1 .
$$

Although $L$ is best determined by numerical experimentation, a useful guide to its value when calculating the $j$ th root can be obtained by use of Stirling's approximation for $L$ !. From

$$
\frac{\left(r_{j} / 2\right)^{2 L+n+1}}{(L+n+1) !(L !)} \sim(10)^{-m}
$$

where $m$ is some pre-selected positive integer, it follows that

$$
\left[2 \pi 10^{-m} L^{L+\frac{1}{2}}(n+L+1)^{n+L+\frac{3}{2}} e^{-(n+2 L+1)}\right]^{1 /(n+2 L+1)} \sim \frac{r_{j}}{2} \sim\left(j+\frac{n}{2}\right) \frac{\pi}{2} .
$$

For $L \gg 1$, this leads to

$$
L\left(1+\frac{(n+1)}{L}\right)^{\frac{1}{2}\left(1+\frac{(n+2)}{2 L}\right)} \sim \frac{\pi e}{2}\left(j+\frac{n}{2}\right) 10^{m /(n+2 L+1)} .
$$

When $n \ll L$ this equation has the approximate solution

$$
L \sim\left[\frac{\pi e}{2}\left(j+\frac{n}{2}\right)-n-1\right] 10^{m /(n+\pi e\{j+n / 2\})} .
$$

When, for example, $j=18, n=5$, and $m=16, L \sim 100$. Table (2) compares the approximate values obtained for the eighteenth root of $J_{5}(z)-i J_{6}(z)=0$ when $L$ and $D$ are varied.

2.4. The error in truncating (19) at $k=L$. In the ascending series calculation we solve

$$
\sum_{k=0}^{L} \frac{(\zeta / 2)^{n+2 k}}{k !(k+n) !}\left[1+\frac{(\zeta / 2)}{(k+n+1)}\right]=0
$$

rather than

$$
\sum_{k=0}^{L} \frac{(\zeta / 2)^{n+2 k}}{k !(k+n) !}\left[1+\frac{(\zeta / 2)}{(k+n+1)}\right]=-\varepsilon
$$

where

$$
\varepsilon=\sum_{k=L+1}^{\infty} \frac{(\zeta / 2)^{n+2 k}}{k !(k+n) !}\left[1+\frac{(\zeta / 2)}{(k+n+1)}\right] .
$$

Clearly, we need $|\varepsilon| \ll 1$ near the roots of the equation. Now put $\zeta=r e^{i \theta}, \varepsilon=\varepsilon_{1}+\varepsilon_{2}$ and consider

$$
\varepsilon_{1}=\sum_{k=L+1}^{\infty} \frac{(r / 2)^{n+2 k} e^{(n+2 k) i \theta}}{k !(k+n) !} .
$$


TABLE 2. Converged results for Newton-Raphson iteration of the ascending series representing $J_{5}(z)-i J_{6}(z)=0$ when $L$ and $D$ (see text) are varied.

\begin{tabular}{|c|c|l|l|c|}
\hline$L$ & $D$ & \multicolumn{1}{|c|}{$x_{18}$} & \multicolumn{1}{|c|}{$y_{18}$} & $\frac{(r / 2)^{2 L+6}}{L !(L+6) !}$ \\
\hline 90 & 40 & $64.158482488 .$. & $-1.579869938 .$. & $1.0310^{-8}$ \\
\hline & 45 & $64.158482487 .$. & $-1.579869938 .$. & $1.0310^{-8}$ \\
\hline 94 & 40 & $64.158482444768 .$. & $-1.579869867681 .$. & $1.6710^{-12}$ \\
\hline & 45 & $64.158482444773 .$. & $-1.579869867628 .$. & $1.6910^{-12}$ \\
\hline 98 & 40 & $64.158482444768 .$. & $-1.579869867620 .$. & $1.9710^{-16}$ \\
\hline & 45 & $64.158482444768 .$. & $-1.579869867616 .$. & $1.9810^{-16}$ \\
\hline
\end{tabular}

Since $L \gg 1$,

$$
2 \pi \varepsilon_{1} \sim\left(\frac{r}{2} e^{(1+i \theta)}\right)^{n} \sum_{k=L+1}^{\infty} \frac{\left(\{r / 2\} e^{(1+i \theta)}\right)^{2 k}}{k^{k+1 / 2}(n+k)^{n+k+1 / 2}}=2 \pi \varepsilon_{1}^{a}, \text { say }
$$

Hence

$$
\begin{aligned}
2 \pi\left|\varepsilon_{1}^{a}\right| & \leq\left(\frac{r e}{2}\right)^{n} \sum_{k=L+1}^{\infty} \frac{(\{r e\} / 2)^{2 k}}{k^{k+1 / 2}(n+k)^{n+k+1 / 2}} \\
& <\left(\frac{r e}{2}\right)^{n} \frac{1}{(L+1)^{1 / 2}(n+L+1)^{n+1 / 2}} \sum_{k=L+1}^{\infty}\left(\frac{r e}{2(L+1)}\right)^{2 k} \\
& =\frac{(\{r e\} / 2)^{n}}{(L+1)^{1 / 2}(n+L+1)^{n+1 / 2}} \frac{\mu^{(L+1)}}{(1-\mu)}
\end{aligned}
$$

where

$$
\mu=\left(\frac{r e}{2(L+1)}\right)^{2} .
$$

From this result the corresponding approximation for $\left|\varepsilon_{2}\right|$ follows at once and, from $|\varepsilon| \leq\left|\varepsilon_{1}\right|+\left|\varepsilon_{2}\right|$, we then obtain $\varepsilon<\varepsilon_{a}$, where

$$
\varepsilon_{a} \sim \frac{1}{2 \pi}\left(\frac{r e}{2}\right)^{n} \frac{1}{(L+1)^{1 / 2}} \frac{\mu^{(L+1)}}{(1-\mu)}\left[\frac{1}{(n+L+1)^{n+1 / 2}}+\frac{(r e)}{2(n+L+2)^{n+3 / 2}}\right] .
$$

In the case of the eighteenth root of $I_{5}(\zeta)+I_{6}(\zeta)=0$, the approximation $r \sim \pi(j+n / 2)$ which, for $j=18$ and $n=5$, is in error by about one third of one percent-and the choice $L=98$ leads to

$$
\varepsilon_{a} \sim 1.41 \times 10^{-13}
$$

which serves as an upper bound for the truncation error in this case. 
3. Results. Tables (3), (4), (5), (6), and (7) compare the first thirty approximate roots of $J_{n}(\zeta)-i J_{n+1}(\zeta)=0$ as calculated from the asymptotic formulae and the asymptotic calculation in each of the five cases corresponding to $n=0,1,2,3$, and 5 , respectively.

Note that the last figure of each root is not rounded. Save for table (3), which lists the first twenty roots, the tables also include the first eighteen roots as calculated by the ascending series approach. Further roots have not been calculated by this approach due to the growth of rounding error as $\left|\zeta_{j}\right|$ increases. The tables show that the accuracy of the asymptotic formulae deteriorates as $n$ increases. This is not surprising since the formulae have been derived on the assumption that $n=O(1)$.

TABLE 3. Unrounded approximate values for the first thirty, fourthquadrant roots, $x+i y$, of $J_{0}-i J_{1}=0$.

\begin{tabular}{|c|c|c|c|c|c|}
\hline \multicolumn{2}{|c|}{ Asymptotic formula } & \multicolumn{2}{|c|}{ Asymptotic calculation } & \multicolumn{2}{|c|}{ Ascending series calculation } \\
\hline 2.979 & -1.282 & 2.9776 & -1.2792 & $2 . 9 8 0 \longdiv { 3 8 2 4 1 4 7 9 0 }$ & -1.279602540299 \\
\hline 6.175104 & -1.6191 & 6.175108 & -1.61868 & 6.175153070954 & -1.618717384471 \\
\hline 9.341952 & -1.81899 & 9.341957 & -1.8188692 & 9.341960983461 & -1.818872787772 \\
\hline 12.498504 & -1.96150 & 12.4985065 & -1.9614588 & 12.498507063958 & -1.961459538019 \\
\hline 15.6501037 & -2.07233 & 15.65010425 & -2.07230961 & 15.650104385309 & -2.072309817830 \\
\hline 18.7989114 & -2.163022 & 18.798911644 & -2.163010912 & 18.798911683696 & -2.163010983274 \\
\hline 21.94597993 & -2.239779 & 21.945979970 & -2.239772463 & 21.945979984381 & -2.239772492276 \\
\hline 25.09188576 & -2.3063169 & 25.091885758 & -2.306312793 & 25.091885763907 & -2.306312806675 \\
\hline 28.23697316 & -2.3650387 & 28.236973142 & -2.365036113 & 28.236973145397 & -2.365036120661 \\
\hline 31.38146101 & -2.4175887 & 31.3814609883 & -2.417586982 & 31.381460989647 & -2.417586986362 \\
\hline 34.52549555 & -2.4651412 & 34.5254955236 & -2.465140068 & 34.525495524330 & -2.465140070798 \\
\hline 37.66917840 & -2.5085645 & 37.66917838219 & -2.5085636976 & 37.669178382576 & -2.508563698916 \\
\hline 40.81258254 & -2.5485185 & 40.81258252502 & -2.5485179035 & 40.812582525250 & -2.548517904322 \\
\hline 43.95576178 & -2.5855165 & 43.95576176345 & -2.5855161403 & 43.955761763589 & -2.585516140857 \\
\hline 47.09875671 & -2.6199660 & 47.09875670430 & -2.6199657071 & 47.098756704391 & -2.619965707516 \\
\hline 50.241598611 & -2.6521953 & 50.24159859851 & -2.6521951319 & 50.241598598574 & -2.652195132137 \\
\hline 53.384311921 & -2.6824734 & 53.384311910838 & -2.68247325772 & 53.384311910875 & -2.682473257885 \\
\hline 56.526916091 & -2.71102300 & 56.526916082180 & -2.711022877648 & 56.526916082206 & -2.711022877758 \\
\hline 59.669426774 & -2.73803078 & 59.669426766956 & -2.738030685256 & 59.669426766973 & -2.738030685336 \\
\hline 62.811856726 & -2.76365475 & 62.811856719871 & -2.763654675022 & 62.811856719883 & -2.763654675081 \\
\hline 65.954216449 & -2.78802979 & 65.954216443289 & -2.788029738475 & & \\
\hline 69.096514672 & -2.81127200 & 69.096514667607 & -2.811271959593 & & \\
\hline 72.238758717 & -2.83348198 & 72.238758713036 & -2.8333481956161 & & \\
\hline 75.380954769 & -2.85474753 & 75.380954765712 & -2.854747510576 & & \\
\hline 78.523108094 & -2.87514568 & 78.523108091021 & -2.875145664058 & & \\
\hline 81.665223203 & -2.894744411 & 81.665223200231 & -2.894744400454 & & \\
\hline 84.807303984 & -2.913604019 & 84.807303982006 & -2.913604012522 & & \\
\hline 87.949353809 & -2.931778224 & 87.949353807156 & -2.931778219939 & & \\
\hline 91.0913756149 & -2.9493150930 & 91.091375612811 & -2.949315091274 & & \\
\hline 94.2333719724 & -2.96625780968 & 94.233371970601 & -2.966257809784 & & \\
\hline
\end{tabular}


For $n>0$, Eqs. (16) and (17) indicate roots for values of $j \leq 0$. When, for example, $n=5$, roots are indicated for $j=0$ and $j=-1$. Insertion of these "roots" as initial approximations for the ascending series calculation results in the successive NewtonRaphson iterations converging to zero. This provides numerical verification of the earlier conjecture that the asymptotic approximation to the $j$ th root of $F_{n}(z)=0$ given by Eqs. (16) and (17) is valid for $p \leq-n-1-j$, where $j \geq 1$ (see Eq. (12)).

TABLE 4. Unrounded approximate values for the first thirty, fourthquadrant roots, $x+i y$, of $J_{1}-i J_{2}=0$.

\begin{tabular}{|c|c|c|c|c|c|}
\hline \multicolumn{2}{|c|}{ Asymptotic formula } & \multicolumn{2}{|c|}{ Asymptotic calculation } & \multicolumn{2}{|c|}{ Ascending series calculation } \\
\hline 4.4204 & -0.926 & 4.4200 & -0.9331 & 4.419943205884 & -0.932937042896 \\
\hline 7.66598 & -1.1827 & 7.666005 & -1183743 & 7.666002709895 & -1.183736098455 \\
\hline 10.854359 & -1.3487 & 10.8543757 & -1.3490255 & 10.854375397749 & -1.349024657437 \\
\hline 14.023113 & -1.47286 & 14.02312174 & -1.4729426 & 14.023121673932 & -1.472942493036 \\
\hline 17.182627 & -1.57214 & 17.18263170 & -1.57217770 & 17.182631682732 & -1.572177640669 \\
\hline 20.337003 & -1.654951 & 20.337006290 & -1.654963656 & 20.337006284216 & -1.654963633544 \\
\hline 23.4882107 & -1.725984 & 23.488212297 & -1.725989485 & 23.488212295270 & -1.725989476180 \\
\hline 26.6373166 & -1.7881833 & 26.6373176099 & -1.788184860 & 26.637317608878 & -1.788184855930 \\
\hline 29.7849540 & -1.8435041 & 29.7849546682 & -1.843504286 & 29.784954667687 & -1.843504283703 \\
\hline 32.9315225 & -1.8933178 & 32.9315230189 & -1.8933173327 & 32.931523018650 & -1.893317331424 \\
\hline 36.0772877 & -1.9386219 & 36.0772880365 & -1.9386311377 & 36.077288036420 & -1.938621136988 \\
\hline 39.2224329 & -1.9801651 & 39.22243317799 & -1.9801643341 & 39.222433177909 & -1.980164333738 \\
\hline 42.3670893 & -2.0185241 & 42.36708949424 & -2.0185233462 & 42.367089494191 & -2.018523345918 \\
\hline 45.51135307 & -2.0541522 & 45.51135319605 & -2.0541514711 & 45.511353196026 & -2.054151470962 \\
\hline 48.65529647 & -2.0874123 & 48.65529657275 & -2.0874116289 & 48.655296572733 & -2.087411628814 \\
\hline 51.79897495 & -2.1185995 & 51.798975032111 & -2.11859889711 & 51.798975032097 & -2.118598897033 \\
\hline 54.94243172 & -2.1479570 & 54.942431783315 & -2.147956431921 & 54.942431783306 & -2.147956431861 \\
\hline 58.08570099 & -2.1756875 & 58.085701037287 & -2.175686976475 & 58.085701037280 & -2.175686976432 \\
\hline 61.22881020 & -2.2019618 & 61.228810246011 & -2.201961347271 & & \\
\hline 64.37178167 & -2.2269252 & 64.371781702377 & -2.226924803728 & & \\
\hline 67.51463368 & -2.2507022 & 67.514633704458 & -2.250701904556 & & \\
\hline 70.65738139 & -2.2734006 & 70.657381416943 & -2.273400262795 & & \\
\hline 73.800037501 & -2.2951137 & 73.800037518047 & -2.295113486295 & & \\
\hline 76.942612677 & -2.31592378 & 76.942612691908 & -2.315923506869 & & \\
\hline 80.085115996 & -2.33590269 & 80.085116007999 & -2.335902444565 & & \\
\hline 83.227555206 & -2.35511433 & 83.227555216753 & -2.355114114137 & & \\
\hline 86.369936973 & -2.37361545 & 86.369936982298 & -2.373615253076 & & \\
\hline 89.512267059 & -2.39145671 & 89.512267067405 & -2.391456530756 & & \\
\hline 92.654550475 & -2.40868355 & 92.654550481752 & -2.408683383912 & & \\
\hline 95.796791596 & -2.42533686 & 95.796791601746 & -2.425336713113 & & \\
\hline
\end{tabular}


TABLE 5. Unrounded approximate values for the first thirty, fourthquadrant roots, $x+i y$, of $J_{2}-i J_{3}=0$.

\begin{tabular}{|c|c|c|c|c|c|}
\hline \multicolumn{2}{|c|}{ Asymptotic formula } & \multicolumn{2}{|c|}{ Asymptotic calculation } & \multicolumn{2}{|c|}{ Ascending series calculation } \\
\hline 5.722 & -0.803 & 5.716100 & -0.81912 & 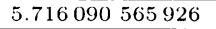 & -0.819169034174 \\
\hline 9.0545 & -1.017 & 9.05380269 & -1.021671 & 9.053802767071 & -1.021674260831 \\
\hline 12.2868 & -1.1614 & 12.28665879 & -1.1630623 & 12.286658838815 & -1.163062837072 \\
\hline 15.48220 & -1.2719 & 15.48215848 & -1.27269998 & 15.482158497264 & -1.272700122514 \\
\hline 18.65965 & -1.3620 & 18.659639189 & -1.36245498 & 18.659639195322 & -1.362455033267 \\
\hline 21.826958 & -1.4382 & 21.826952051 & -1.438499621 & 21.826952054080 & -1.438499639187 \\
\hline 24.987934 & -1.50436 & 24.987932188 & -1.504492147 & 24.987932189059 & -1.504492154786 \\
\hline 28.1446891 & -1.56270 & 28.1446881664 & -1.562789835 & 28.144688166963 & -1.562789838819 \\
\hline 31.2984802 & -1.61494 & 31.2984798744 & -1.615004118 & 31.298479874715 & -1.615004120129 \\
\hline 34.45010844 & -1.66224 & 34.45010835840 & -1.6622866019 & 34.450108358544 & -1.662286603090 \\
\hline 37.60010813 & -1.70546 & 37.60010815567 & -1.7054894055 & 37.600108155755 & -1.705489406252 \\
\hline 40.74884999 & -1.74524 & 40.74885006572 & -1.7452609904 & 40.748850065769 & -1.745260990875 \\
\hline 43.89659954 & -1.78209 & 43.89659962865 & -1.7821064003 & 43.896599628686 & -1.782106400630 \\
\hline 47.04355205 & -1.81641 & 47.04355214084 & -1.8164267156 & 47.043552140861 & -1.816426715830 \\
\hline 50.18985444 & -1.848536 & 50.18985452617 & -1.8485457904 & 50.189854526191 & -1.848545790586 \\
\hline 53.33561942 & -1.878721 & 53.335619492862 & -1.87872890211 & 53.335619492870 & -1.878728902192 \\
\hline 56.48093491 & -1.907190 & 56.480934980481 & -1.90719608513 & 56.480934980487 & -1.907196085192 \\
\hline 59.62587057 & -1.934127 & 59.625870632976 & -1.934131872115 & 59.625870632980 & -1.934131872158 \\
\hline 62.77048228 & -1.959688 & 62.770482337485 & -1.959692544872 & & \\
\hline 65.91481543 & -1.984008 & 65.914815472593 & -1.984011623167 & & \\
\hline 69.05890723 & -2.007201 & 69.058907275529 & -2.007204081512 & & \\
\hline 72.20278856 & -2.029367 & 72.202788595564 & -2.029369632311 & & \\
\hline 75.34648518 & -2.050593 & 75.346485211925 & -2.050595313009 & & \\
\hline 78.49001881 & -2.070956 & 78.490018837612 & -2.070957547185 & & \\
\hline 81.63340787 & -2.090522 & 81.633407893270 & -2.090523802969 & & \\
\hline 84.77666809 & -2.1093528 & 84.776668110411 & -2.109353939673 & & \\
\hline 87.91981299 & -2.1275003 & 87.919813006398 & -2.127501310444 & & \\
\hline 91.06285424 & -2.1450128 & 91.062854261979 & -2.145013672134 & & \\
\hline 94.20580201 & -2.1619332 & 94.205802023938 & -2.161933941483 & & \\
\hline 97.348665138 & -2.1783002 & 97.348665149693 & -2.178300827747 & & \\
\hline
\end{tabular}

TABLE 6 . Unrounded approximate values for the first thirty, fourthquadrant roots, $x+i y$, of $J_{3}-i J_{4}=0$.

\begin{tabular}{|c|c|c|c|c|c|}
\hline \multicolumn{2}{|c|}{ Asymptotic formula } & \multicolumn{2}{|c|}{ Asymptotic calculation } & \multicolumn{2}{|c|}{ Ascending series calculation } \\
\hline 6.976 & -0.736 & 6.953081 & -0.759105 & 6.953093480994 & -0.759096845464 \\
\hline 10.389 & -0.922 & 10.3855661 & -0.931074 & 10.385556842568 & -0.931073031515 \\
\hline 13.6703 & -1.051 & 13.66940957 & -1.0553837 & 13.669409657684 & -1.055383532845 \\
\hline 16.8978 & -1.152 & 16.89747658 & -1.15402850 & 16.897476598033 & -1.154028436512 \\
\hline 20.0978 & -1.2350 & 20.097723115 & -1.23611376 & 20.097723119991 & -1.236113783692 \\
\hline 23.28196 & -1.3058 & 23.281902130 & -1.30650760 & 23.281902131961 & -1.306507593797 \\
\hline 26.45593 & -1.3677 & 26.4559017084 & -1.368167987 & 26.455901708892 & -1.368167982700 \\
\hline 29.62304 & -1.4227 & 29.6230222597 & -1.423041774 & 29.623022259944 & -1.423041772018 \\
\hline 32.785276 & -1.4722 & 32.78526599442 & -1.472483874 & 32.785265994499 & -1.472483873080 \\
\hline 35.943928 & -1.5173 & 35.94392159519 & -1.5174773541 & 35.943921595228 & -1.517477353390 \\
\hline 39.099861 & -1.5586 & 39.09985766081 & -1.5587590840 & 39.099857660830 & -1.558759083554 \\
\hline 42.253684 & -1.59681 & 42.253681767980 & -1.5968960849 & 42.253681767986 & -1.596896084694 \\
\hline 45.405833 & -1.63227 & 45.405832085642 & -1.6323342447 & 45.405832085645 & -1.632334244602 \\
\hline 48.556634 & -1.66538 & 48.556632804572 & -1.6654306560 & 48.556632804573 & -1.665430655952 \\
\hline 51.7063299 & -1.69643 & 51.706329072015 & -1.6964757970 & 51.706329072016 & -1.696475796963 \\
\hline 54.8551104 & -1.72567 & 54.855109781857 & -1.72570917129 & 54.855109781858 & -1.725709171236 \\
\hline 58.0031233 & -1.75330 & 58.003122887876 & -1.753330599881 & 58.003122887876 & -1.753330599838 \\
\hline 61.1504863 & -1.77948 & 61.150485960012 & -1.779508543603 & 61.150485960012 & -1.779508543572 \\
\hline 64.2972938 & -1.80437 & 64.297293626497 & -1.804386348098 & & \\
\hline 67.4436231 & -1.82807 & 67.443622925577 & -1.828087005572 & & \\
\hline 70.5895373 & -1.85070 & 70.589537222545 & -1.850716837538 & & \\
\hline 73.7350892 & -1.87235 & 73.735089122433 & -1.872368379678 & & \\
\hline 76.88032275 & -1.893114 & 76.880322667006 & -1.893122667928 & & \\
\hline 80.02527507 & -1.913043 & 80.025275013519 & -1.913051069144 & & \\
\hline 83.16997778 & -1.932210 & 83.169977732668 & -1.932216761155 & & \\
\hline 86.31445786 & -1.950670 & 86.314457823023 & -1.950675939890 & & \\
\hline 89.45873854 & -1.968474 & 89.458738511751 & -1.968478811867 & & \\
\hline 92.60283991 & -1.985666 & 92.602839892458 & -1.985670416294 & & \\
\hline 95.74677945 & -2.002287 & 95.746779437588 & -2.002291310751 & & \\
\hline 98.89057242 & -2.018374 & $98.890572+13293$ & -2.018378146745 & & \\
\hline
\end{tabular}


TABLE 7. Unrounded approximate values for the first thirty, fourthquadrant roots, $x+i y$, of $J_{5}-i J_{6}=0$.

\begin{tabular}{|l|l|l|l|l|l|}
\hline \multicolumn{2}{|c|}{ Asymptotic formula } & \multicolumn{2}{|c|}{ Asymptotic calculation } & \multicolumn{2}{c|}{ Ascending series calculation } \\
\hline 9.41 & -0.66 & 9.3329 & -0.69445 & 9.333119629036 & -0.694442717434 \\
\hline 12.964 & -0.813 & 12.9450 & -0.829375 & 12.945103691825 & -0.829366713388 \\
\hline 16.341 & -0.922 & 16.334615 & -0.930806 & 16.334617793373 & -0.930803342054 \\
\hline 19.6370 & -1.008 & 19.6342921 & -1.0136782 & 19.634292825814 & -1.013677175727 \\
\hline 22.8881 & -1.081 & 22.8867958 & -1.0841896 & 22.886796022166 & -1.084189240232 \\
\hline 26.1116 & -1.143 & 26.11097873 & -1.1457220 & 26.110978807715 & -1.145721839349 \\
\hline 29.3170 & -1.198 & 29.316667198 & -1.20038044 & 29.316667230893 & -1.200380333030 \\
\hline 32.5097 & -1.2485 & 32.509549134 & -1.24958404 & 32.509549148968 & -1.249583980947 \\
\hline 35.6933 & -1.2936 & 35.693168309 & -1.29434278 & 35.693168316421 & -1.294342747257 \\
\hline 38.86995 & -1.3348 & 38.869857307 & -1.33540439 & 38.869857311224 & -1.335404369643 \\
\hline 42.04128 & -1.3729 & 42.041218668 & -1.373339596 & 42.041218670385 & -1.373339583208 \\
\hline 45.20843 & -1.4082 & 45.2083919675 & -1.408594768 & 45.208391968773 & -1.408594759819 \\
\hline 48.37224 & -1.4412 & 48.3722109023 & -1.441526063 & 48.372210903072 & -1.441526057709 \\
\hline 51.53332 & -1.4722 & 51.5333001125 & -1.472422463 & 51.533300113022 & -1.472422459933 \\
\hline 54.69215 & -1.5013 & 54.6921372210 & -1.501521825 & 54.692137221329 & -1.501521822656 \\
\hline 57.849106 & -1.5288 & 57.8490939107 & -1.529022359 & 57.849093910987 & -1.529022357589 \\
\hline 61.004473 & -1.5549 & 61.0044638971 & -1.555091038 & 61.004463897257 & -1.555091036904 \\
\hline 64.158489 & -1.5797 & 64.15848244468 & -1.579869868 & 64.158482444768 & -1.579869867616 \\
\hline 67.311346 & -1.60340 & 67.31134027888 & -1.603480657 & & \\
\hline 70.463198 & -1.62596 & 70.46319369056 & -1.626028685 & & \\
\hline 73.614175 & -1.64754 & 73.61417199984 & -1.647605577 & & \\
\hline 76.764386 & -1.66824 & 76.76438315325 & -1.668291577 & & \\
\hline 79.913920 & -1.68811 & 79.91391797839 & -1.688157357 & & \\
\hline 83.062855 & -1.70722 & 83.06285345842 & -1.707265491 & & \\
\hline 86.211256 & -1.72563 & 86.21125528061 & -1.725671646 & & \\
\hline 89.359181 & -1.74339 & 89.35917984057 & -1.743425564 & & \\
\hline 92.506676 & -1.76054 & 92.50667583332 & -1.760571878 & & \\
\hline 95.6537864 & -1.77712 & 95.65378552754 & -1.777150788 & & \\
\hline 98.8005465 & -1.79317 & 98.80054579435 & -1.793198629 & & \\
\hline 101.9469896 & -1.808730 & 101.94698894420 & -1.808748353 & & \\
\hline
\end{tabular}

\section{REFERENCES}

[1] S. Tadepalli and C. E. Synolakis, Roots of $J_{\gamma}(z) \pm i J_{\gamma+1}(z)=0$ and the evaluation of integrals with cylindrical function kernals, Quart. Appl. Math. LII, 103-111 (1994)

[2] G. N. Watson, Theory of Bessel Functions, Cambridge University Press, 1944

[3] D. A. MacDonald, The roots of $J_{0}(z)-i J_{1}(z)$, Quart. Appl. Math. XLVII, 375-378 (1989)

[4] M. Renardy, Problems and Solutions, Ed. M. Klamkin, Siam Review, vol. 31, 1989, pp. 126-127 